\title{
A Survey on Vehicle Detection based on Vision
}

\author{
Surender Dhanasekaran ${ }^{1}$, Karthi Ramachandran ${ }^{1}$, Selvamuthukumar $\mathrm{M}^{2}$, Nithya Velam $^{3} \&$ Soma Pal ${ }^{3}$ \\ ${ }^{1}$ Assistant Professor, EEE Department, School of Electrical \& Computing, Vel Tech University, India \\ ${ }^{2}$ Assistant Professor, Automobile Department, School of Mechanical \& Construction Engineering, Vel Tech \\ University, India \\ ${ }^{3}$ ECE Department, School of Electrical \& Computing Vel Tech University, Chennai, India \\ Correspondence: Surender Dhanasekaran, Assistant Professor, EEE Department, School of Electrical \& \\ Computing, Vel Tech University, India. E-mail: surenderdhanasekaran@gmail.com
}

Received: July 4, 2015

doi:10.5539/mas.v9n12p118
Accepted: August 13, 2015

Online Published: October 31, 2015

URL: http://dx.doi.org/10.5539/mas.v9n12p118

\begin{abstract}
Passenger safety in vehicles is the primary concern and hence Vehicle detection for providing enough safety information for Driver Assistant System (DAS) become popular among researchers. Many approaches use different types of information such as shadow, edge, light, to detect the vehicles. Here vision based vehicle detection techniques were summarized. Following topics discussed below, camera placement and the various applications of monocular vehicle detection, common features and common classification methods, motionbased approaches and nighttime vehicle detection and monocular pose estimation.
\end{abstract}

Keywords: vehicle detection, DAS, ITS, vision based detection, tracking.

\section{Introduction}

Nowadays thousands of drivers and passengers were losing their lives every year on road accident, due to deadly crashes between more than one vehicles. To save lives and reduce the number of on-road fatalities the Research and development putting much efforts in advanced sensing, environmental perception, and IDA systems. There are number of many research focuses were dedicated to the development of intellectual driver assistance systems and autonomous vehicles over the past decade, which reduces the danger by monitoring the on-road environment. In particular, researchers attracted towards the on-road detection of vehicles in recent years [1]. Different methods were available for on-road vehicle detection which includes radar, LIDAR, and computer vision. In recent years imaging technology has immensely progressed. Computing power has dramatically increased. Concurrently, Cameras are cheaper, smaller, and of higher quality than ever before. Furthermore, parallelization, such as multicore processing and graphical processing units (GPUs), of computing platforms geared in recent years. Such hardware advances allow real-time implementation for vehicle detection using computer vision approaches. With advances in computational technologies and camera sensing, advances in vehicle detection using monocular vision, stereo vision, and sensor fusion with vision have been an extremely active research area in the intelligent vehicles community. Also extensive study has been done on On-road vehicle tracking. Detect and track on-road vehicles in real time now become commonplace for research studies to report the ability to reliably over extended periods [2]. The aggregate of this spatiotemporal information from vehicle detection and tracking can be used to identify maneuvers and to learn, model, and classify on-road behavior.

Use of vision for on-road interpretation is shows in Figure 1. At the left end, cues were used for on-road vehicle detection listed as motion and appearance etc. One level towards right, detected vehicles are associated across frames, allowing for vehicle tracking. Dynamic motion of detected vehicles measured in Vehicle tracking. At the right end, an aggregate of spatiotemporal features allows for characterization of vehicle behavior, recognition of specific maneuvers, behavior classification, and long-term motion prediction. Examples of work in this emerging area used for prediction of turning behavior, [4] has prediction of lane changes, and [3] has modeling typical on-road behavior. In this paper, various works of vision-based vehicle detection techniques has been reviewed. 


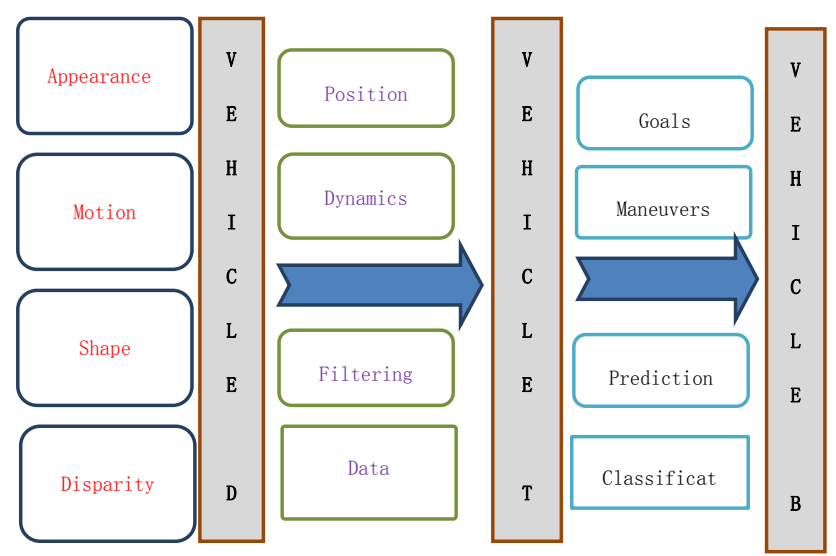

Figure 1. Vehicle detection and Tracking steps

\section{Vision-Based Vehicle Detection}

With the help of one or more cameras and some cues shown in left end of Figure 1, vehicles are detected. Countless challenges exist in computer vision stand-point, on-road vehicle detection. Only weak assumptions are made about the scene structure in on-road environment. The system needs to detect, recognize, and localize the object in video to detect from a moving platform often without dependence on background modeling. Effects of ego and relative motion are introduced by the vehicles on the road in motion. Size, shape, and color of vehicles are variable in the road [1]. Vehicle features has variations in illumination, background, and scene complexity in on-road environment. Erroneous detections can be introduced by complex shadowing, man-made structures, and ubiquitous visual clutter. Many orientations are also encountered in vehicles, including preceding, oncoming, and cross traffic. Scene clutter leads to partial occlusion of vehicle by limiting the full visibility of vehicles in on-road environment. Furthermore, 100\% accuracy needed in order to provide the human or autonomous driver with advanced notice of critical situations a vehicle detection system needs to operate at real-time speeds.

Following topics discussed below, camera placement and the various applications of monocular vehicle detection, common features and common classification methods, motion- based approaches and nighttime vehicle detection and monocular pose estimation

\subsection{Camera Placement}

With respect to the ego vehicle a single camera aims to detect vehicles in a variety of locations. To detect preceding and oncoming vehicles the vast majority of monocular vehicle detection studies position the camera looking forward. Valuable insight and safety-critical applications yielded by various novel camera placements. To monitor vehicle's blind spot the camera attached on the side-view mirror, facing toward the rear of the vehicle. Because of the field of view and high inconsistency in the look of vehicles, depending on their relative positions detecting vehicles became difficult with this camera placement. Some of the camera placement positions and detections methods were reviewed below.

\subsubsection{Front Facing}

Following work have focused on detecting the forego vehicles by placing the camera front focusing. To detect overtaking vehicles using optical flow algorithm a monocular camera placed within the car [5]. When multiple targets found then one which is near to the source car alone will focus and tracked. Kalman filter were used for vehicle tracking. Blind spot also detected using optical flow; also both cars and two wheelers on the lateral side of a driving vehicle can be detected in [6]. In [7], to monitor the blind spot area a camera is mounted on the vehicle. Full panoramic view of the on-road scene was acquired by mounting an omnidirectional camera on top of the vehicle. Two kinds of part-based features are combined they are related to the characteristics of the vehicle, and multiple models were constructed based on different viewpoints of a vehicle. To construct the detector and estimate the reasonable position of the presence of the vehicle the location information of each feature is incorporated. In $[8]$, to estimate the vehicle's 
ego motion, detecting static objects using optical flow the omnidirectional vision was used. Using Kalman filtering moving objects were also detected and tracked over long periods of time. While passing the front vehicle to generate a path, a new path planning method introduced that satisfies the vehicle non holonomic constraint. Finally calculate the safe lane change distance for autonomous vehicle [9]. Normalized cross-correlation method and centroid-area-difference are used for Vehicles detection. The movements of the vehicle are tracked by headlights. The vehicle speed estimate with the help of pin-hole and Euclidean distance methods [10] from the fixed-camera the video sequence have been obtained for analysis. Using telescopic virtual coil movements such as left turn, go straight, turn right of the each vehicle on the road lane, and are detected.

\subsubsection{Side Mirrors}

In order to detect the overtaking vehicles camera has been place in side mirrors. In [11], Blind spot vehicle detection is presented, using video data obtained from the both left and right mirror of a car, proposed system detects cars in the particular configuration of blind spot, and one side video is used for edge detection and support vector machine (SVM) learning and on the other side is used for template matching.. In [12], window-based tracking is employed. Vehicles in nearby lanes are detected by first detecting the front wheel and then the rear wheel using a camera looking out the side passenger's window. The Kalman filter is used for tracking the combined parts. In [13], the camera was similarly attached on the side of the TerraMax autonomous experimental vehicle test-bed. Two high resolution wide angle cameras are used in this, with respect of the moving direction each one looking laterally (70 degrees), with an adaptive background subtraction based technique, and motion cues were used to detect vehicles in the side view along with speed estimation and tracking.

\subsubsection{Back Facing}

Some of the researchers were shown interest to detect the following vehicle by placing the camera on the back side. In [14], out of the rear windshield the camera was positioned looking backward. While changing lane the following vehicles front faces was detected, in order to advise the driver on the safety. A region of interest was generated using symmetry and edge operator. Haar wavelet feature extraction used to detect the vehicles and SVM classification.

\subsection{Appearance-Features}

For detecting vehicles a variety of appearance features have been used. By measuring the symmetry of an image patch about a vertical axis in the image plane to detect vehicle many earlier works uses this local symmetry operators.

\subsubsection{Edge, Shadow and Symmetry}

Features like edge, shadow and symmetry used for detecting the vehicles in the following works. Often, after evaluating edge operators over the image the symmetry was computed on image patches, to recognize the vertical sides of the rear face of a vehicle [15]. Including street surface information, shadow and symmetry features are abstracted to 3D sensors and combined with two system models with an cooperating multiple model filter, one system models used for constant velocity, another system models used for constant acceleration. Information of the shape and symmetry of the vehicle and the shadow were used to define geometric model of the vehicle. Algorithm had been speed up with help of a multi-resolution approach. Edge information helps highlight the sides of the vehicle, as well as its cast shadow. Underneath, vertical edge, symmetry and taillight these four cues are merged for the forgoing vehicle detection. The processes including initial sampling, propagation, observation and cue fusion and evaluation are completed by using particle filter with four cues; also particle filter accurately generates the vehicle distribution [16]. To detect vehicles at nighttime symmetry was used along with detected circular headlights and edge energy in [17]. A preceding vehicle was tracked and detected by includes road area finding, vehicle footprint extraction, and vehicle bounding box extraction. To avoid the false detection the footprint of the vehicle inside the road area was detected. Vehicles effectively tracked by extracting the preceding vehicle, and detect the passing car and distant car. Symmetry and edges were also used in [18]. A monochrome images captured by a single camera mounted inside a vehicle and mainly been focused on daylight conditions vehicle detection and tracking. It includes road area to avoid false detections of vehicles caused by the distraction of background objects. Using "shadow" as a cue vehicle presence inside the road area is hypothesized and tracked using a Kalman filter in [19].

In recent years, general and robust feature sets are used for detecting vehicle rather than from simpler image features like edges and symmetry. These feature sets allow for direct classification and detection of objects in images, now common in the computer vision literature. 
Features like Histogram of oriented gradient (HOG) and Haar-like are extremely well represented in the vehicle detection literature, as they are in the object detection literature.

\subsubsection{Histogram of Oriented Gradient HOG Features}

First evaluating edge operators over the image used to extract the HOG features and then discretizing and ditching the orientations of the edge intensities into a histogram [20].

HOG features are expressive image features, showing good detection presentation in a range of computer vision tasks. In [21], for vehicle detection the symmetry of the HOG features extracted in a given image patch, along with the HOG features themselves. In some cases vehicle pose are also determined by HOG features. The main drawback of HOG features is that they are quite slow to compute. Implementing HOG feature extraction using GPU, recent works have speeding up the process.

\subsubsection{Haar-Like Features}

In Haar-like features [22] sums and differences of rectangles over an image patch are composed. Haar-like features are well suited for real-time detection of vehicles or vehicle parts due to following advantages such as highly efficient to compute, sensitive to vertical, horizontal and symmetric structures. In [14], with a rear-facing camera the front faces of following vehicles were captured and vehicle was detected by extracting Haar features. Using front-facing camera mostly Haar-like features have been used to detect the rear faces of foregoing vehicles. By detecting the front and rear wheels the Haar-like features are used for detect the side profiles of vehicles [12].Also Haar-like features have been used to track vehicles in the image plane.

\subsubsection{Others Feature based Detection}

In [23], HOG features and Haar-like features were analyzed and they are combined to detect vehicle. To detect the rear faces of vehicles, including during partial occlusions Scale Invariant Feature Transform(SIFT) features were used in [24].The Hidden Random Field (HRF) model extended to incorporate logistic regression classifiers into unary potentials to detect the vehicle in in a still image taken by camera. In [7], SURF: Speeded up Robust Features and edges were combined to detect vehicles in the blind spot. In [25], vehicle has been reconditioned within a region of interest (ROI) in an image by obtaining the ROI by a radar sensor then two classifiers support vector machine (SVM) and a neural network have been used to verify the effectiveness of the features and they are used for vehicle detection. For detecting parked sedans in static images dimensionality reduction of the feature space, using a combination of principal component analysis and independent component analysis, was used in [26].

\subsection{Motion-Based Approaches}

Motion of the vehicles is also used for detecting the vehicles in many approaches following are the works related to vehicle detection using movement.

\subsubsection{Forego Motion}

In [13], an adaptive background model was constructed to differentiated vehicles from the background based on motion. To model the area where overtaking vehicles tend to appear in the camera's field of view an adaptive background modeling was used in [27]. Geometrical relations between the elements in the scene so that moving objects were exploited for vehicle detection. In [28], between the successive images homography matrix was computed; for reliable and accurate homography estimation Kalman filtering based probabilistic framework is presented, which in turn allows to detect the moving vehicles in the image. This method seems likely to return many false alarms, but quantitative performance analysis was not included. Optical flow was used in conjunction with appearance-based techniques. Ego-motion estimation using optical flow and integrated detection of vehicles was implemented in [29]. Ego-motion estimation using an omnidirectional camera and detection of vehicles was implemented in [8].

\subsubsection{Over Taking Motion}

A model of overtaking vehicle areas in the images in the scene background was implemented by integrates dynamic scene modeling, hypothesis testing, and robust information fusion was used in [30]. A combination of optical flow and symmetry tracking was used for vehicle detection also interest points that persisted over long periods of time were detected as vehicles traveling parallel to the ego vehicle. In [5], optical flow was used to detect overtaking vehicles in the blind spot. A similar approach for detecting vehicles in the blind spot was reported in [6]. 


\subsection{Nighttime Vision Based Detection}

The vast majority of vision-based vehicle detection papers are dedicated to daytime conditions. Night-time conditions may be dealt with in a few ways. Some techniques make use of wide dynamic range of cameras to acquire the image or video during night times. In some approaches day time detection method used for detecting vehicles at night time with well-illuminated nighttime [16].

Often by detecting the headlights and taillights of vehicles absent specialized hardware or illumination infrastructure, various studies have trained specific models for detecting vehicles at nighttime, encountered on the road. In low-light conditions color space thresholding used for initial segmentation step in detecting vehicle lights. In [31], using stereo vision by extracting vertical edges and 3-D and color in the Lab color space the vehicle is detected at nighttime. In [30], based on image segmentation and pattern analysis techniques the oncoming and preceding vehicles were detected and tracked, thresholding the grayscale image were used for localizing the taillights. In [17], symmetry, edge energy, and detected circles are used to track vehicles using particle filters. To compute longitudinal distance for nighttime vehicles uses the pinhole model is featured in [32] Vehicles are detected by localizing pairs of red taillights in the hue-saturation-value color space. The camera has been configured to reliably output colors by controlling the exposure, optimizing the appearance of taillights for segmentation. The segmented taillights are detected as pairs using cross correlation and symmetry. Vehicles are then tracked in the image plane using Kalman filtering. In [33], multiple vehicles are detected by tracking headlight and taillight blobs, a detection-by-tracking approach. The tracking problem is formulated as a maximum posterior inference problem over a random Markov field.

\section{Conclusion}

In this paper an important research area of Vehicle detection works had been review. In particular vision based works had been literature here. Previous works on the vehicle detection listed based on camera poisons, feature based detection and motion based detection works and night time detection. Various aspects has been reviewed by camera placement and the various applications of monocular vehicle detection, common features and common classification methods, motion- based approaches and nighttime vehicle detection and monocular pose estimation. In future combined continuous detection can be implemented which includes detecting two wheelers as well as four wheeler.

\section{References}

Arrospide, J., Salgado, L., \& Nieto, M. (2010). Vehicle detection and tracking using homography-based plane rectification and particle filtering, in Proc. IEEE IV, Jun., 150-155.

Aytekin, B., \& Altug, E. (2010). Increasing driving safety with a multiple vehicle detection and tracking system using ongoing vehicle shadow information, inProc. IEEE Int. Conf. SMC, Oct., 3650-3656.

Barth, A., \& Franke, U. (2010). Tracking oncoming and turning vehicles at intersections, in Proc. 13th Int. IEEE ITSC, 861-868.

Blanc, N., Steux, B., \& Hinz, T. (2007). LaRASideCam: A fast and robust vision-based blindspot detection system, inProc. IEEE Intell. Veh.Symp., Jun., 480-485.

Broggi, A., Cappalunga, A., Cattani, S., \& Zani, P. (2008). Lateral vehicles detection using monocular high resolution cameras on TerraMax, in Proc. IEEE Intell. Veh.Symp., 1143-1148.

Cabani, I., Toulminet, G., \& Bensrhair, A. (2005). Color-based detection of vehicle lights, inProc. IEEE Intell. Veh.Symp., Jun., 278-283

Chan, Y. M., Huang, S. S., Fu, L. C., \& Hsiao, P. Y. (2007). Vehicle detection under various lighting conditions by incorporating particle filter, in Proc. IEEE ITSC, 534-539

Chang, H. Y., Fu, C. M., \& Huang, C. L. (2005). Real-time vision-based pre-ceding vehicle tracking and recognition, in Proc. IEEE Intell.Veh.Symp., Jun., 514-519.

Chang, W. C., \& Cho, C. W. (2008). Real-time side vehicle tracking using parts-based boosting, in Proc. IEEE Int. Conf. SMC, 3370-3375.

Chen, J. J., Zhao, P., Mei, T., \& Liang, H. W. (2013). Lane change path planning based on piecewise Bezier curve for autonomous vehicle Vehicular Electronics and Safety (ICVES), 2013 IEEE International Conference, $17-22$.

Chen, Y. L., Lin, C. T., Fan, C. J., Hsieh, C. M., \& Wu, B. F. (2008). Vision-based nighttime vehicle detection and range estimation for driver assis-tance, inProc. IEEE Int. Conf. SMC, Oct., 2988-2993 
Cheon, M., Lee, W., Yoon, C., \& Park, M. (2012). Vision-based vehicle detec-tion system with consideration of the detecting location, IEEE Trans. Intell. Transp. Syst., 13(3), 1243-1252.

Dalal, N., \& Triggs, B. (2005). Histograms of oriented gradients for human detection, inProc. IEEE Comput. Soc. Conf. CVPR, 886-893.

Diaz Alonso, J., Ros Vidal, E., Rotter, A., \& Muhlenberg, M. (2008). Lane-change decision aid system based on motion-driven vehicle tracking, IEEE Trans. Veh. Technol., 57(5), 2736-2746.

Fossati, A., Schönmann, P., \& Fua, P. (2010). Real-time vehicle tracking for driving assistance. Mach. Vis. Appl., 22(2), 439-448.

Gandhi, T., \& Trivedi, M. M. (2005). Parametric ego-motion estimation for vehicle surround analysis using an omnidirectional camera. Mach. Vis. Appl., 16(2), 85-95.

Hoffmann, C. (2006). Fusing multiple 2D visual features for vehicle detection, inProc. IEEE Intell. Veh.Symp, 406-411.

Idler, C., Schweiger, R., Paulus, D., Mahlisch, M., \& Ritter, W. (2006). Real-time vision based multi-target-tracking with particle filters in automotive applications, inProc. IEEE Intell.Veh.Symp., 188-193.

Kasper, D., Weidl, G., Dang, T., Breuel, G., Tamke, A., \& Rosenstiel, W. (2011). Object-oriented Bayesian networks for detection of lane change maneu-vers, inProc. IEEE IV, 673-678.

Lin, B. F., Chan, Y. M., Fu, L. C., Hsiao, P. Y., Chuang, L. A., Huang, S. S., \& Lo, M. F. (2012). Integrating appearance and edge features for sedan vehicle detection in the blind-spot area, IEEE Trans. Intell. Transp. Syst., 13(2), 737-747.

Liu, W., Wen, X., Duan, B., Yuan, H., \& Wang, N. (2007). Rear vehicle detection and tracking for lane change assist, inProc. IEEE Intell. Veh.Symp., 252-257.

Lowe, D. (1999). Object recognition from local scale-invariant features. In Proc. Int. Conf. Comput. Vis., 1150-1157

Malley, R. O., Jones, E., \& Glavin, M. (2010). Rear-lamp vehicle detection and tracking in low-exposure color video for night conditions, IEEE Trans. Intell. Transp. Syst., 11(2), 453-462.

Negri, P., Clady, X., Hanif, S. M., \& Prevost, L. (2008). A cascade of boosted generative and discriminative classifiers for vehicle detection, EURASIP J. Adv. Signal Process., 136.

Sina, I., Wibisono, A., Nurhadiyatna, A., Hardjono, B., Jatmiko, W., \& Mursanto, P. (2013). Vehicle counting and speed measurement using headlight detection, Advanced Computer Science and Information Systems (ICACSIS), IEEE Conference Publications, 149-154.

Sivaraman, S., Morris, B. T., \& Trivedi, M. M. (2011). Learning multi-lane trajectories using vehicle-based vision, in Proc. IEEE Int. Conf. Compute. Vision Workshop, 2070-2076.

Song, K. T., \& Chen, H. Y. (2007). Lateral driving assistance using optical flow and scene analysis, inProc. IEEE Intell. Veh.Symp., Jun., 624-629.

Sun, Z., Bebis, G., \& Miller, R. (2006). On-road vehicle detection: A review, IEEE Trans. Pattern Anal. Mach. Intell., 28(5), 694-711.

Viola, P., \& Jones, M. (2001). Rapid object detection using a boosted cascadeof simple features, inProc. IEEE Comput. Vis. Pattern Recog. Conf., I-511-I-518.

Wang, C. C. R., \& Lien, J. J. (2008). Automatic vehicle detection using local features: A statistical approach, IEEE Trans. Intell. Transp. Syst., 9(1), 83-96.

Wang, J., Bebis, G., \& Miller, R. (2005). Overtaking vehicle detection using dynamic and quasi-static background modeling, inProc. IEEE CVPR, Jun. 64.

Zhang, Y., Kiselewich, S., \& Bauson, W. (2006). Legendre and Gabor moments for vehicle recognition in forward collision warning, in Proc. IEEE ITSC, Sep., 1185-1190

Zhu, Y., Comaniciu, D., Pellkofer, M., \& Koehler, T. (2006). Reliable detection of overtaking vehicles using robust information fusion, IEEE Trans. Intell. Transp. Syst., 7(4), 401-414.

\section{Copyrights}


Copyright for this article is retained by the author(s), with first publication rights granted to the journal.

This is an open-access article distributed under the terms and conditions of the Creative Commons Attribution license (http://creativecommons.org/licenses/by/3.0/). 